\title{
Thickness dependence of the anomalous Hall effect in thin films of the topological semimetal $\mathrm{Co}_{2} \mathrm{MnGa}$
}

\author{
Anastasios Markou, ${ }^{1, *}$ Dominik Kriegner, ${ }^{1}$ Jacob Gayles, ${ }^{1}$ Liguo Zhang, ${ }^{1}$ Yi-Cheng Chen,,${ }^{1,2}$ \\ Benedikt Ernst, ${ }^{1}$ Yu-Hong Lai, ${ }^{2}$ Walter Schnelle, ${ }^{1}$ Ying-Hao Chu, ${ }^{2}$ Yan Sun, ${ }^{1}$ and Claudia Felser ${ }^{1}$ \\ ${ }^{1}$ Max Planck Institute for Chemical Physics of Solids, Nöthnitzer Str. 40, 01187 Dresden, Germany \\ ${ }^{2}$ Department of Materials Science and Engineering, \\ National Chiao Tung University, 30010 Hsinchu, Taiwan
}

(Dated: August 9, 2019)

\begin{abstract}
Topological magnetic semimetals promise large Berry curvature through the distribution of the topological Weyl nodes or nodal lines and further novel physics with exotic transport phenomena. We present a systematic study of the structural and magnetotransport properties of $\mathrm{Co}_{2} \mathrm{MnGa}_{\text {films }}$ from thin $(20 \mathrm{~nm})$ to bulk like behavior $(80 \mathrm{~nm})$, in order to understand the underlying mechanisms and the role on the topology. The magnetron sputtered $\mathrm{Co}_{2} \mathrm{MnGa}$ films are $L 2_{1}$-ordered showing very good heteroepitaxy and a strain-induced tetragonal distortion. The anomalous Hall conductivity was found to be maximum at a value of $1138 \mathrm{~S} / \mathrm{cm}$, with a corresponding anomalous Hall angle of $13 \%$, which is comparatively larger than topologically trivial metals. There is a good agreement between the theoretical calculations and the Hall conductivity observed for the $80 \mathrm{~nm}$ film, which suggest that the effect is intrinsic. Thus, the $\mathrm{Co}_{2} \mathrm{MnGa}$ compound manifests as a promising material towards topologically-driven spintronic applications.
\end{abstract}

\section{INTRODUCTION}

The experimental realization of graphene, characterized by a low energy Dirac dispersion, ${ }^{1}$ reinvigorated the interest in utilizing and understanding the topological electronic nature of materials. One such avenue led to the topological insulators determined by the surface Dirac dispersion states and gapped states in the bulk. ${ }^{2}$ Contrary to topological insulators, the exotic topological semimetals are advantageous due to an intimate correlation of the dispersion of the bulk and surface states. The Dirac, ${ }^{3,4}$ Weyl, ${ }^{5,6}$ and nodal line ${ }^{7}$ semimetals are the most well-known examples. The Weyl semimetal (WSM) is found in systems with a lack of inversion symmetry or time reversal symmetry, where two momentum-space distributed Weyl nodes of opposite chirality form and are linked by the so-called Fermi-arc surface bands. ${ }^{8-11}$ The finite Berry curvature associated with the Weyl nodes or gapped nodal line leads to exotic phenomena, such as the chiral anomaly, ${ }^{12-15}$ magnetoptical ${ }^{16}$ and transport responses, ${ }^{17}$ the anomalous Hall effect (AHE), ${ }^{18,19}$ and the Nernst effect counterparts. ${ }^{20-22}$ In magnetic Heusler compounds, this has become a major point of interest, ${ }^{17}$ where large values of the AHE have been associated with the momentum-space distribution of the Weyl nodes and gapped nodal lines.

Recently, Wang et al. ${ }^{23}$ proposed that several Co-based full Heusler compounds realize WSMs. They focused on $\mathrm{Co}_{2} \mathrm{ZrSn}$ compound and found that two Weyl points exist close to the Fermi energy when the magnetization is along the [110] direction. Chang et al. ${ }^{24}$ pinpointed the topological semimetal states in the $\mathrm{Co}_{2} \mathrm{TiZ}(\mathrm{Z}=\mathrm{Si}, \mathrm{Ge}$ or Sn) compounds by first-principle calculations. Kübler and Felser ${ }^{25}$ suggested that the experimentally observed

\footnotetext{
* Anastasios.Markou@cpfs.mpg.de
}

large $\mathrm{AHE}$ in $\mathrm{Co}_{2} \mathrm{MnAl}$ films ${ }^{26}$ is due to the distribution of four Weyl points just above the Fermi edge, while they, also, predicted similar results for the $\mathrm{Co}_{2} \mathrm{MnGa}$ compound. Further, it was found to be a nodal line and Weyl semimetal. ${ }^{27}$

The bulk $\mathrm{Co}_{2} \mathrm{MnGa}$ Heusler compound crystallizes in the cubic $\mathrm{Cu}_{2} \mathrm{MnAl}$-type structure with space group $F m \overline{3} m$ (No. 225 ) and lattice constant $a=5.77 \AA$. The Co atoms occupy the Wyckoff position $8 c$, whereas the $\mathrm{Mn}$ and $\mathrm{Ga}$ occupy the positions $4 b$ and $4 a$, respectively. It is a semimetallic ferromagnet with a large saturated magnetic moment of $M_{\mathrm{s}}=4.05 \mu_{\mathrm{B}} /$ f.u., following the Slater Pauling rule, ${ }^{28}$ and high Curie temperature of $T_{\mathrm{C}}=694 \mathrm{~K}^{29,30}$ Single crystals of $\mathrm{Co}_{2} \mathrm{MnGa}$ were found to exhibit large $\mathrm{AHE}^{31}$ and anomalous Nernst effect (ANE), ${ }^{22,32}$ which originates from the large Berry curvature distribution around Fermi energy and associated with nodal lines ${ }^{27,33}$ or Weyl points. ${ }^{25}$ Albeit, for device applications a proper understanding of the thin film limit is required. There have been some studies on thin films of $\mathrm{Co}_{2} \mathrm{MnGa}$, in particular, a $\mathrm{MgO} / \mathrm{Co}_{2} \mathrm{MnGa} / \mathrm{Pd}$ stack with perpendicular magnetic anisotropy that shows $\mathrm{AHE}^{34}$ and $\mathrm{Co}_{2} \mathrm{MnGa}$ films with one of the largest values reported for the ANE. ${ }^{35}$ To fully utilize the topological properties, one must understand the dependence of the transport properties from the bulk-like films to the thin film limit. In the thin film limit, the properties of the surface play a significant role due to the intrinsic topology rooted in the electronic structure.

In this work, we present the structural, magnetic and transport properties of high-quality $\mathrm{Co}_{2} \mathrm{MnGa}$ films with thickness ranging from 20 to $80 \mathrm{~nm}$. We performed systematic x-ray diffraction (XRD), transmission electron microscopy (TEM), magnetic and transport characterization of films heteroepitaxially grown on $\mathrm{MgO}$ substrates. We find that the magnetotransport properties vary with the temperature and the film thickness. A strong AHE is observed in the $80 \mathrm{~nm}$ thick film that agrees with bulk 

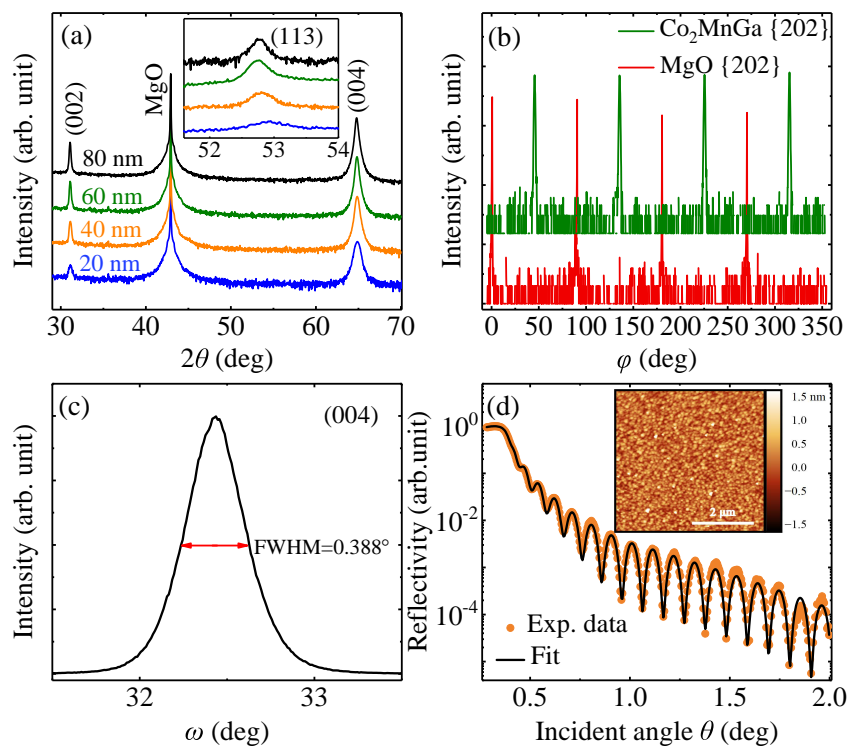

Figure 1. (a) XRD pattern of the $\mathrm{Co}_{2} \mathrm{MnGa}$ films with different thickness $(20-80 \mathrm{~nm})$. The inset shows the (113) assymetric reflections. (b) $\varphi$-scan patterns of the $\{202\}$ planes from

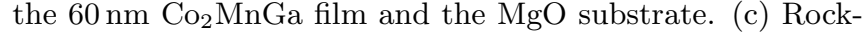
ing curve ( $\omega$-scan) of (004) reflection from a $80 \mathrm{~nm} \mathrm{Co}_{2} \mathrm{MnGa}$. (d) X-ray reflectivity pattern of the $40 \mathrm{~nm} \mathrm{Co}_{2} \mathrm{MnGa}$, where the solid line represents the least-squares fit to the data.

first-principle calculations of the intrinsic AHE. Lastly, the $80 \mathrm{~nm}$ film shows to be the upper limit for both the AHE $(1138 \mathrm{~S} / \mathrm{cm})$ and anomalous Hall angle (AHA) of $13 \%$, while the $20 \mathrm{~nm}$ film displays an AHE of $840 \mathrm{~S} / \mathrm{cm}$ and an AHA of $10 \%$.

\section{EXPERIMENTAL DETAILS}

$\mathrm{Co}_{2} \mathrm{MnGa}$ films with thicknesses of 20,40,60 and $80 \mathrm{~nm}$ have been grown heteroepitaxially on $\mathrm{MgO}(001)$ single crystal substrates. A BESTEC UHV magnetron sputtering system was used for the deposition of the films, with $\mathrm{Co}(5.08 \mathrm{~cm}), \mathrm{Mn}(5.08 \mathrm{~cm})$ and $\mathrm{Mn}_{50} \mathrm{Ga}_{50}(5.08 \mathrm{~cm})$ sources in confocal geometry. The target to substrate distance was $20 \mathrm{~cm}$. Prior to deposition, the chamber was evacuated to a base pressure less than $8 \times 10^{-9}$ mbar, while the process gas $(\operatorname{Ar} 5 \mathrm{~N})$ pressure was $3 \times 10^{-3}$ mbar. The $\mathrm{Co}_{2} \mathrm{MnGa}$ films were grown by co-sputtering and the individual sputter rates were adjusted to obtain the desired composition. The Co was deposited by applying $34 \mathrm{~W}$ dc power, the Mn by applying $6 \mathrm{~W}$ dc power and the $\mathrm{Mn}_{50} \mathrm{Ga}_{50}$ by applying $22 \mathrm{~W} \mathrm{dc}$, and the total rate was $0.58 \AA / \mathrm{s}$. The substrates were rotated during deposition, to ensure homogeneous growth. The films were grown at $550^{\circ} \mathrm{C}$ and then post-annealed in situ for an additional 20 minutes to improve the chemical ordering. All samples were capped with a $3 \mathrm{~nm}$ thick $\mathrm{Al}$ film at room temperature to prevent oxidation.

Stoichiometry was estimated as $\mathrm{Co}_{51} \mathrm{Mn}_{25} \mathrm{Ga}_{24}$ by energy-dispersive x-ray spectroscopy (EDXS) with an ex-
TABLE I. Lattice parameters, volume, tetragonallity $c / a$ and the full width at half maximum (FWHM) of the (004) rocking curve profile for $\mathrm{Co}_{2} \mathrm{MnGa}$ thin films with different thickness.

\begin{tabular}{cccccc}
\hline \hline $\begin{array}{c}\text { Thickness } \\
(\mathrm{nm})\end{array}$ & $\begin{array}{c}c \\
(\AA)\end{array}$ & $\begin{array}{c}a \\
(\AA)\end{array}$ & $\begin{array}{c}\text { Volume } \\
\left(\AA^{3}\right)\end{array}$ & $c / a$ & $\begin{array}{c}\text { FWHM } \\
(\mathrm{deg})\end{array}$ \\
\hline 20 & 5.728 & 5.811 & 193.4 & 0.986 & 0.495 \\
40 & 5.741 & 5.793 & 192.7 & 0.991 & 0.485 \\
60 & 5.751 & 5.785 & 192.4 & 0.994 & 0.424 \\
80 & 5.747 & 5.786 & 192.4 & 0.993 & 0.388 \\
\hline \hline
\end{tabular}

perimental uncertainty of $2 \%$. X-ray diffraction (XRD) and $\mathrm{x}$-ray reflectivity (XRR) were measured with a Panalytical X'Pert ${ }^{3}$ MRD diffractometer, using $\mathrm{CuK}_{\alpha 1}$ radiation $(\lambda=1.5406 \AA)$. The growth rates and the film thicknesses were determined by a quartz crystal microbalance and confirmed by using XRR measurements. The atomic force microscopy (AFM) image was obtained with a MFP-3D Origin ${ }^{+}$microscope from Oxford Instruments Asylum Research. High-resolution scanning transmission electron microscopy (HRSTEM) was performed using a JEOL ARM200F microscope operated at $200 \mathrm{kV}$. Additionally, the JEOL was equipped with EDXS for element mapping with high spatial resolution. Diffraction patterns were collected by a FEI Tecnai operated at $200 \mathrm{kV}$. Cross-section samples were prepared by focused ion beam milling (FIB). A protective C-Pt layer was deposited on the stack before starting the cross-section preparation. Magnetization measurements were carried out using a Quantum Design (MPMS3 SQUID-VSM) magnetometer and the transport measurements were carried out on bars with a rectangular shape $(7 \mathrm{~mm} \times 2 \mathrm{~mm})$ in a sixprobe method using bonded $\mathrm{Al}$ wires contacts with lowfrequency alternating current (ETO, PPMS9 Quantum Design).

\section{RESULTS AND DISCUSSION}

\section{A. Structural properties}

Different x-ray scattering measurements, $2 \theta-\omega$ scan, rocking curve ( $\omega$-scan) and sample azimuth $(\varphi)$ scan, were performed to study the structure, the crystallinity and the heteroepitaxial relationship between the films and the substrate, respectively. The in-plane lattice mismatch between the $45^{\circ}$ rotated $\mathrm{MgO}$ unit cell $\left(\sqrt{2} a_{\mathrm{MgO}}\right)$ and bulk $\mathrm{Co}_{2} \mathrm{MnGa}$ is $3.2 \%$, which allows the heteroepitaxial growth of (001) oriented films. Fig. 1(a) shows the XRD patterns of the 20,40,60 and $80 \mathrm{~nm} \mathrm{C_{2 }} \mathrm{MnGa}$ films. In addition to the (002) reflection of the $\mathrm{MgO}$ substrate, all the samples exhibit exclusively the (002) and (004) reflections of the cubic $\mathrm{Co}_{2} \mathrm{MnGa}$, indicating (001)-oriented films.

The properties of the Heusler compounds are strongly dependent on the occupation of the crystallographic sites. To determine if the films are fully chemically ordered in 
$L 2_{1}$-type, in addition to (002) reflection, the presence of the superstructure (111) or (113) reflections are needed. The inset in Fig. 1(a) shows $2 \theta-\omega$ scans of the asymmetric (113) Bragg reflection of the $\mathrm{Co}_{2} \mathrm{MnGa}$ thin films. The data were acquired in coplanar diffraction geometry with a linear detector and the acquisition software integrates along the rocking angle $(\omega)$. Quantitative analysis of the integrated intensities for all Bragg reflections, considering the diffraction geometry, as well as polarization, Lorentz, and absorption corrections ${ }^{36}$ consistently showed that below $10 \%$ of $B 2$-type disorder is present in the films, which therefore can be considered almost fully chemically ordered.

$\varphi$-scan patterns of the $\{202\}$ planes from the $\mathrm{Co}_{2} \mathrm{MnGa}$ film and the $\mathrm{MgO}$ substrate are depicted in Fig. 1(b). The reflections of $\mathrm{Co}_{2} \mathrm{MnGa}$ show four-fold symmetry with $90^{\circ}$ intervals, suggesting single crystalline epilayers with well-defined in-plane orientation. By comparing the diffractions of film and substrate a $45^{\circ}$ inplane rotation of the $\mathrm{Co}_{2} \mathrm{MnGa}$ unit cell is observed with respect to the $\mathrm{MgO}$ substrate. The crystallographic orientation relationship is thus determined as $\mathrm{Co}_{2} \mathrm{MnGa}(001)[110] \| \mathrm{MgO}(001)[100]$.

The crystal quality of the $\mathrm{Co}_{2} \mathrm{MnGa}$ films was evaluated from the FWHM values of rocking curves measured around (004) reflection. The small FWHM $\approx 0.388^{\circ}$ (Fig. 1(c)) suggests that the $80 \mathrm{~nm}$ film shows high crystalline quality with low mosaicity. The FWHM remains below $0.5^{\circ}$ for the thinner films indicating a very good crystal quality (Table I).

In spite of the high-temperature growth, all the films are very smooth. Fig. 1(d) shows the XRR pattern of the $40 \mathrm{~nm} \mathrm{Co}_{2} \mathrm{MnGa}$ film with $3 \mathrm{~nm} \mathrm{Al}$ capping layer. The thickness oscillations that start after the critical angle up to $2 \mathrm{deg}$ and beyond indicate smooth films and sharp interfaces. From the good agreement between the experimental data (data points) and the model curve calculated using a modified Parrat formalism ${ }^{37}$ (solid line), we deduce the structural parameters, such as thickness, roughness, and density. Thickness and density agree within $2 \%$ with the nominal values and the determined substrate/film and film/capping layer roughnesses are found to be 3.4 and $4.5 \AA$, respectively. The smooth surface topography is confirmed with AFM from a $40 \mathrm{~nm}$ thick film without Al capping layer, as depicted in the inset of Fig. 1(d). The film is continuous and very smooth with a root mean square roughness of $3 \AA$, which is in good agreement with the XRR measurement.

In order to understand how the strain-induced by the substrate influences the $\mathrm{Co}_{2} \mathrm{MnGa}$ films, we performed reciprocal space map measurements around the (004) and (224) Bragg reflections of $\mathrm{Co}_{2} \mathrm{MnGa}$. Fig. 2 shows that for all film thicknesses, the defined peaks are observed for both Bragg peaks, which indicates the epitaxial growth for all thicknesses. The peak position varies, which reflects a change of the lattice parameters with the film thickness. The extracted lattice parameters are summarized in Table I and show that the in-plane lattice parameter slightly decreases with thickness, while simulta-

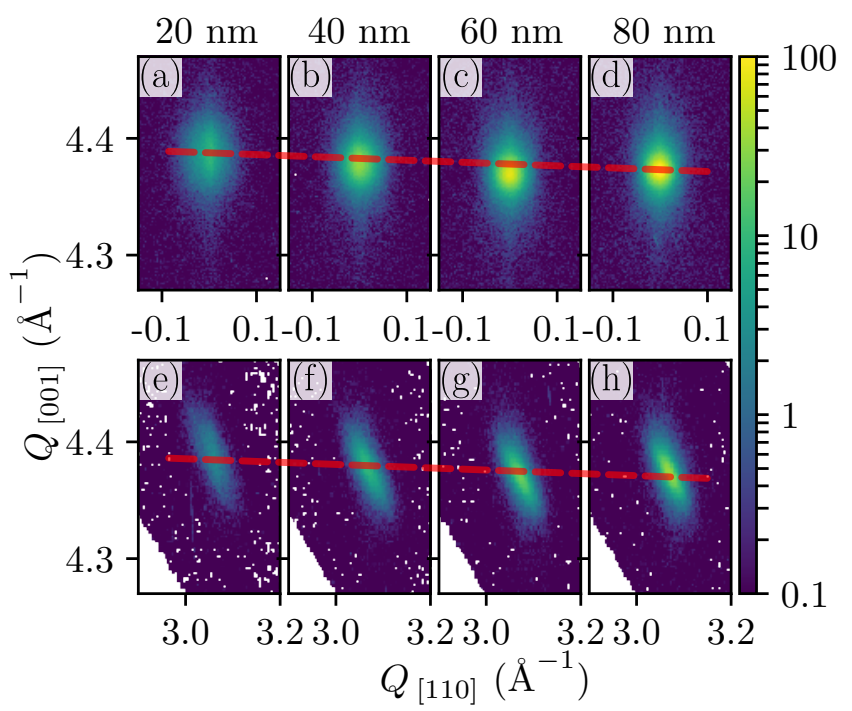

Figure 2. X-ray diffraction reciprocal space maps of $\mathrm{Co}_{2} \mathrm{MnGa}$ thin films. Panels (a)-(e) and (f)-(j) show the diffracted intensity on a logarithmic color scale in the vicinity of the (004) and (224) Bragg peaks of the films, respectively. The $x$ and $y$ axis of the plot show the momentum transfer $Q$ along the [110] and and [001] directions defined as $Q_{[110]}=4 \pi / \lambda \sin \theta \sin (\omega-\theta)$, and $Q_{[001]}=$ $4 \pi / \lambda \sin \theta \cos (\omega-\theta) . \quad \lambda=1.5406 \AA$ is the wavelength of the $\mathrm{x}-$ ray radiation. Red lines are guides to the eye and show that with increasing film thickness the peak position is changing.

neously the out-of-plane parameter increases, hence the unit cell volume remains almost constant. The thinner films display a tetragonal distortion, while thicker films approach the crystalline cubic structure found in bulk compounds. Given the reported lattice parameters of our films in Table I, we conclude that the effective symmetry of the films is reduced to space group $I 4 / \mathrm{mmm}$ (No. 139).

\section{B. TEM investigation}

TEM was performed in the $80 \mathrm{~nm}$ thick $\mathrm{Co}_{2} \mathrm{MnGa}$ to evaluate the film quality on the nanoscale. In Fig. 3(a) we show the cross-section HRSTEM image of the film. The high-quality growth of the $\mathrm{Co}_{2} \mathrm{MnGa}$ film on $\mathrm{MgO}$ substrate is manifested by the crystal lattice of the $\mathrm{Co}_{2} \mathrm{MnGa}$ film. Despite the lattice mismatch of $2.9 \%$ between film and substrate, the $\mathrm{Co}_{2} \mathrm{MnGa}$ epilayer is characterized by good heteroepitaxy with no significant defects. Furthermore, the interface between film and substrate is very sharp with atomic-level flatness.

We depict the selected area electron diffraction (SAED) pattern of the same sample in Fig. 3(b), where the electron beam is parallel to the [110] zone axis of the $\mathrm{MgO}$ substrate. The red and blue open circles correspond to the diffraction spots from the $\mathrm{MgO}$ substrate and the $\mathrm{Co}_{2} \mathrm{MnGa}$ film, respectively. The two different sets of diffraction spots are aligned, confirming the heteroepitaxial growth of the epilayer on the substrate. 

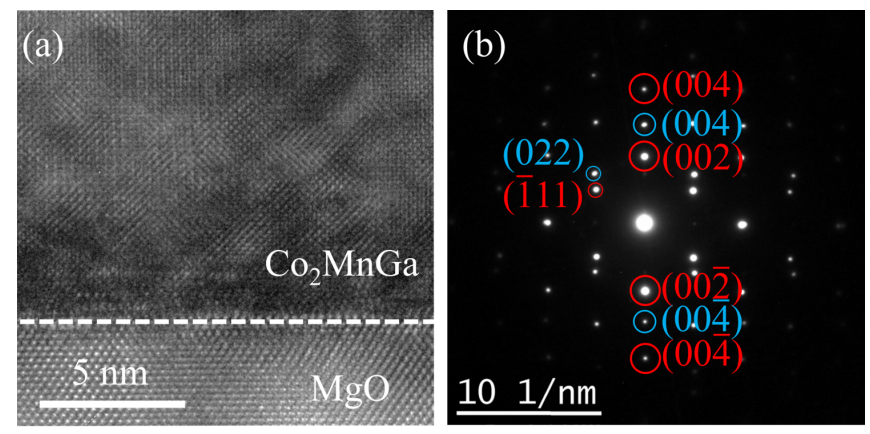

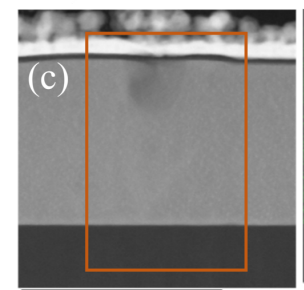

$100 \mathrm{~nm}$

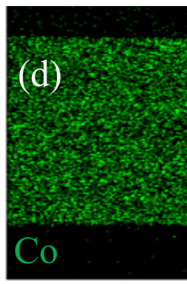

$50 \mathrm{~nm}$

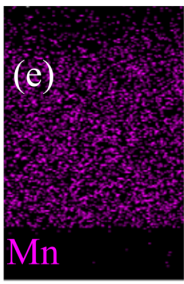

$50 \mathrm{~nm}$

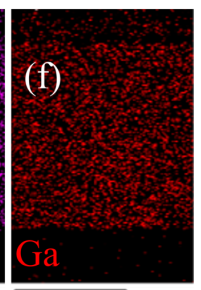

$50 \mathrm{~nm}$
Figure 3. (a) Cross-section HRSTEM image of the $80 \mathrm{~nm}$ $\mathrm{Co}_{2} \mathrm{MnGa}$ film grown on $\mathrm{MgO}$ substrate. (b) SAED pattern showing the diffraction spots from $\mathrm{Co}_{2} \mathrm{MnGa}$ (blue open circles) and $\mathrm{MgO}$ (red open circles). (c) Cross-section HAADFSTEM image, where the orange box denotes the area, where chemical mapping was performed. Elemental mapping of $(\mathrm{d})$ Co (green), (e) Mn (purple) and (f) Ga (red).

Fig. 3(c) shows the high-angle annular dark-field (HAADF) STEM image, where from bottom to top the $\mathrm{MgO}$ substrate, the $\mathrm{Co}_{2} \mathrm{MnGa}$ film and the $\mathrm{Al}$ capping layer are clearly shown in different brightness. The film is continuous, smooth and with the expected thickness of $80 \mathrm{~nm}$. The element distribution within the $\mathrm{Co}_{2} \mathrm{MnGa}$ films was analyzed by EDXS element mapping and the orange box in the HAADF-STEM image (Fig. 3(c)) indicates the area where the elemental analysis was carried out. The spatial distribution of the count rate intensity of the $\mathrm{Co}, \mathrm{Mn}$, and Ga elements are represented with different colors in Fig. 3(d)-(f). All elements were detected at exactly the same sample regions, therefore confirming the homogeneity of the $\mathrm{Co}_{2} \mathrm{MnGa}$.

\section{Magnetic and transport properties}

In Fig. 4 we show typical in-plane and out-of-plane magnetization hysteresis loops for the $80 \mathrm{~nm} \mathrm{Co}_{2} \mathrm{MnGa}$ film measured at $300 \mathrm{~K}$. The $\mathrm{Co}_{2} \mathrm{MnGa}$ film is a soft magnet with an in-plane magnetic easy axis. The saturation magnetization is $M_{\mathrm{S}}=760 \mathrm{kA} / \mathrm{m}$ and the coercivity is $\mu_{0} H_{\mathrm{c}}=0.5 \mathrm{mT}$. The Curie temperature was measured using SQUID magnetometry with an oven option. The magnetization was recorded with a constant in-plane field of $\mu_{0} H=50 \mathrm{mT}$ while warming up the sample, as shown in the inset of Fig. 4. The curve is normalized at $400 \mathrm{~K}$ and the extracted Curie temperature is $700 \pm 5 \mathrm{~K}$. The saturation magnetization and the Curie temperature $\left(M_{\mathrm{s}}\right.$ $=4.20 \mu_{\mathrm{B}} / \mathrm{f}$.u. and $T_{\mathrm{C}}=700 \mathrm{~K}$ )are slightly higher com-

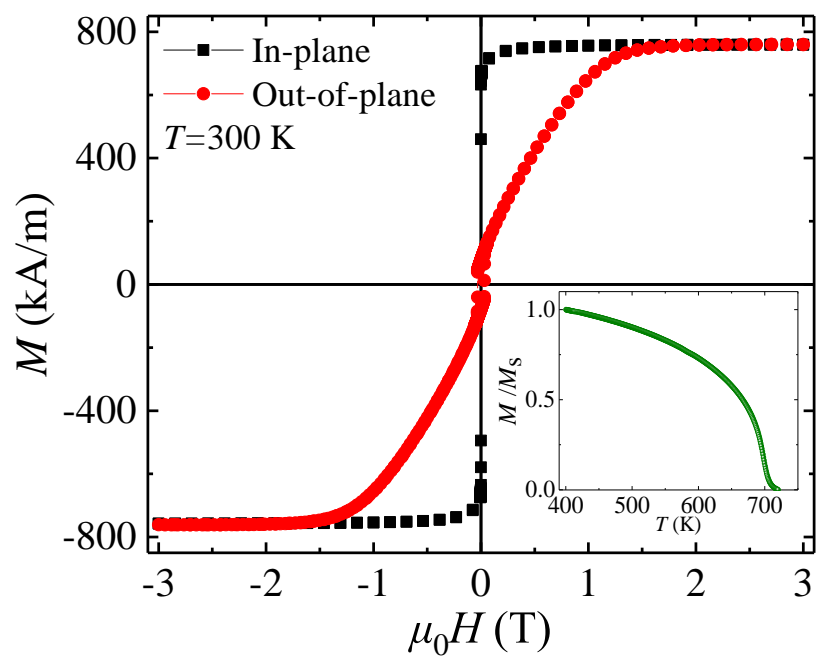

Figure 4. In-plane and out-of-plane magnetization hysteresis loops of the $80 \mathrm{~nm} \mathrm{Co} 2 \mathrm{MnGa}$ film. The inset shows the temperature dependence of normalized magnetization for the $60 \mathrm{~nm} \mathrm{Co}_{2} \mathrm{MnGa}$ film.

pared to the bulk ${ }^{29}\left(M_{\mathrm{s}}=4.05 \mu_{\mathrm{B}} /\right.$ f.u. and $\left.T_{\mathrm{C}}=694 \mathrm{~K}\right)$ and the calculated ${ }^{28}\left(M_{\mathrm{S}}=4.058 \mu_{\mathrm{B}} /\right.$ f.u. $)$ values. This can be attributed to the small amount of disorder and/or off-stoichiometry in our films and it is also in a good agreement with electronic structure calculations, which take into account disorder in $\mathrm{Mn} / \mathrm{Ga}$ sites. ${ }^{38}$

We show the transport properties of the $\mathrm{Co}_{2} \mathrm{MnGa}$ films in Fig. 5. The transport measurements are collected with the magnetic field and the current applied along the [001] and [110] directions of the $\mathrm{Co}_{2} \mathrm{MnGa}$, respectively. Fig. 5(a) depicts the temperature dependent resistivity at zero field for the $80 \mathrm{~nm} \mathrm{Co} 2 \mathrm{MnGa}$ film. The film shows metallic behavior with a residual resistivity of $110 \mu \Omega \mathrm{cm}$ at $40 \mathrm{~K}$. Above $45 \mathrm{~K}, \rho_{\mathrm{xx}}(T)$ is nonlinear, which indicates mixed phonon and magnon scattering state. ${ }^{39}$ The upturn of longitudinal resistivity below $45 \mathrm{~K}$ is attributed to defects or due to diffusion channel electron-electron interaction mechanism as recently reported in $L 2_{1}-\mathrm{Co}_{2} \mathrm{MnAl}$ films. ${ }^{40}$ The inset in Fig. 5(a) shows the residual resistivity ratio $\left(\mathrm{RRR}=\rho_{300 \mathrm{~K}} / \rho_{40 \mathrm{~K}}\right)$ as a function of film thickness. The RRR increases linearly as the thickness increases and reaches 1.17 for the $80 \mathrm{~nm}$ film, which compares well with other Co-based full Heusler compound films. ${ }^{40-42}$

In Fig. 5(b) the first quadrant of the Hall resistivity loops are shown for the $80 \mathrm{~nm}$ and the $20 \mathrm{~nm}$ at $T=300$, 75,5 and $2 \mathrm{~K}$. We analyze the Hall resistivity in the saturated state by using

$$
\rho_{\mathrm{yx}}=R_{0} \mu_{0} H+\rho_{\mathrm{yx}}^{\mathrm{AHE}},
$$

where $\mu_{0}, R_{0}$ and $\rho_{\mathrm{yx}}^{\mathrm{AHE}}=R_{\mathrm{S}} M$ are the permeability of free space, the ordinary Hall coefficient, $R_{\mathrm{S}}$ is the anomalous Hall coefficient and $M$ is the magnetization perpendicular to the plane of the film, respectively. There is a clear trend that shows a saturated Hall resistivity above 

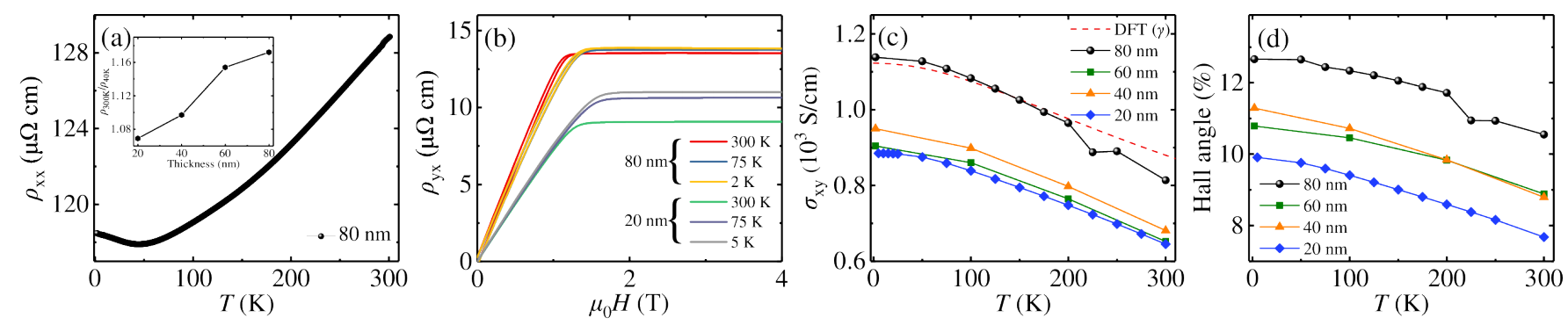

Figure 5. (a) Temperature dependent longitudinal resistivity of the $80 \mathrm{~nm} \mathrm{Co}_{2} \mathrm{MnGa}$ film. The inset shows the thickness dependent RRR. (b) Hall resistivity as a function of the applied magnetic field for the $80 \mathrm{~nm}$ and $20 \mathrm{~nm} \mathrm{Co}_{2} \mathrm{MnGa}_{\mathrm{Gilms}}$ collected at 300, 75, 5 and $2 \mathrm{~K}$. (c) Temperature dependent Hall conductivity of $\mathrm{Co}_{2} \mathrm{MnGa}$ films with different thickness. The red dashed line represents the constant $\gamma$ broadening from ab-initio calculations. (d) Temperature dependent anomalous Hall angle of $\mathrm{Co}_{2} \mathrm{MnGa}$ films with different thickness.

applied fields of $2 \mathrm{~T}$ for all thicknesses and temperatures. The tetragonal distortion of the $\mathrm{Co}_{2} \mathrm{MnGa}$ films is also reflected in the Hall resistivity, where the $80 \mathrm{~nm}$ film, which approaches the cubic structure, saturates close to an applied field of $1.2 \mathrm{~T}$ and the more tetragonally distorted $20 \mathrm{~nm}$ film at $1.5 \mathrm{~T}$. Furthermore, the magnitude of the AHE varies with the thickness and the temperature, where it is maximized at $2 \mathrm{~K}$. The maximum and minimum of the Hall resistivity is found to be $\rho_{\mathrm{yx}}=14 \mu \Omega \mathrm{cm}$, and $11 \mu \Omega \mathrm{cm}$ for the 80 and $20 \mathrm{~nm}$ films, respectively. In order to compare our experimental results with theoretical calculations, we extract the Hall conductivity as a function of temperature.

The experimental values of the anomalous Hall conductivity $\left(\sigma_{\mathrm{xy}}^{\mathrm{AHE}}\right)$ are estimated by the Hall resistivity $\rho_{\mathrm{yx}}$ and the longitudinal resistivity as:

$$
\sigma_{\mathrm{xy}}^{\mathrm{AHE}}=\frac{\rho_{\mathrm{yx}}}{\rho_{\mathrm{yx}}^{2}+\rho_{\mathrm{xx}}^{2}} .
$$

In Fig 5(c) the temperature dependence of $\sigma_{\mathrm{xy}}^{\mathrm{AHE}}$ for all films are compared to the theoretical calculations. For all films, the largest $\sigma_{\mathrm{xy}}^{\mathrm{AHE}}$ is observed at the lowest temperature, and is gradually reduced with increasing temperature. The thicker sample $(80 \mathrm{~nm})$ shows a large value of $\sigma_{\mathrm{xy}}^{\mathrm{AHE}}=1138 \mathrm{~S} / \mathrm{cm}$ at $2 \mathrm{~K}$ and $\sigma_{\mathrm{xy}}^{\mathrm{AHE}}=814 \mathrm{~S} / \mathrm{cm}$ at $300 \mathrm{~K}$, which are consistent with the bulk values, ${ }^{22}$ while in thinner samples the $\sigma_{\mathrm{xy}}^{\mathrm{AHE}}$ is reduced.

We calculate the AHE in the bulk $\mathrm{Co}_{2} \mathrm{MnGa}$ compound using interpolated tight-binding Hamiltonian on a $351^{3} \mathbf{k}$-grid from the first-principle electronic structure converged on a $13^{3} \mathbf{k}$-grid from the VASP code. ${ }^{43}$ The AHE is calculated using the Kubo formula within the constant $\gamma$ broadening approximation: ${ }^{18,44}$

$$
\begin{aligned}
\sigma_{i j}^{\mathrm{AHE}} & \stackrel{\gamma \rightarrow 0}{=} \\
& \frac{2 e^{2} \hbar}{\mathcal{N}} \sum_{\mathbf{k}, n}^{\mathrm{occ}} \sum_{m \neq n} \operatorname{Im}\left[\frac{\left\langle\psi_{\mathbf{k}, n}\left|v_{i}\right| \psi_{\mathbf{k}, m}\right\rangle\left\langle\psi_{\mathbf{k}, m}\left|v_{j}\right| \psi_{\mathbf{k}, n}\right\rangle}{\left(\mathcal{E}_{\mathbf{k}, m}-\mathcal{E}_{\mathbf{k}, n}\right)^{2}+\gamma^{2}}\right] .
\end{aligned}
$$

Here, $v_{i}=\frac{1}{\hbar} \frac{\partial H}{\partial k_{i}}$ is the canonical velocity operator, and $\left|\psi_{\mathbf{k}, n}\right\rangle$ is the eigenstate of $H$ with eigenvalue $\mathcal{E}_{\mathbf{k}, n}$. The $\gamma$ dependence of the AHE in Fig. 5(c) shows an excellent agreement with the experimental results of the $80 \mathrm{~nm}$ film. This suggests that the $80 \mathrm{~nm}$ film is sufficient to reproduce the bulk properties of $\mathrm{Co}_{2} \mathrm{MnGa}$ and that the anomalous Hall conductivity is due to the intrinsic mechanisms. It is important to note that Eq. 3 does not take into account side-jump and skew-scattering contributions to the AHE which can play a crucial role in films. ${ }^{45-47}$ However, the experimental results show a decrease in the AHE with the decrease of the film thickness and with the same temperature dependence. This constant shift of the AHE with thickness variation suggest an increase of side-jump mechanism that has a similar temperature dependence as the intrinsic Berry curvature. ${ }^{18}$ The side-jump is opposite in sign to the intrinsic mechanism and may be due to the $B 2$-type disorder or the increased role of the surfaces as the thickness is decreased. Since, the B2-type disorder is also present in bulk $\mathrm{Co}_{2} \mathrm{MnGa}$ compounds, ${ }^{29,30}$ therefore we attribute the side-jump to the increased role of the surfaces. The skew-scattering contribution nominally displays a strong variation with temperature, due to the linear dependence on the longitudinal resistivity. ${ }^{18}$ Furthermore, there may be phonon-assisted skew-scattering effects that show intrinsic behavior which cannot be disentangled in the current ab-initio theory. ${ }^{48}$ The Weyl nodes and nodal lines are primarily due to the spin-up channel which leads to a suppression of spin scattering ${ }^{22}$ analogous to the case in ferromagnetic half-metals. A recent work of $\mathrm{MgO} / \mathrm{Co}_{2} \mathrm{MnGa} / \mathrm{Pd}$ stacks $^{34}$ shows longitudinal resistivity $\sim 30 \mu \Omega \mathrm{cm}$ smaller and the AHE is an order of magnitude smaller $\left(\mathrm{MgO} / \mathrm{Co}_{2} \mathrm{MnGa} / \mathrm{Pd}\right.$ stack: $\sigma_{\mathrm{xy}}^{\mathrm{AHE}} \approx 125 \mathrm{~S} / \mathrm{cm}$ ), compared to our films. This work claimed all three AHE mechanisms to be present, however dominated by the intrinsic and side-jump mechanisms.

The anomalous Hall angle $\theta_{\mathrm{H}}=\sigma_{\mathrm{xy}}^{\mathrm{AHE}} / \sigma_{\mathrm{xx}}$ reflects the ability of a material to deviate the electron flow from the direction of the longitudinal electric field, due to the anomalous Hall effect. The temperature dependence of Hall angle for all $\mathrm{Co}_{2} \mathrm{MnGa}$ films is shown in Fig. 5(d). We find that the AHA varies with the temperature and the film thickness in a similar way as the temperature 
dependence of the Hall conductivity. The AHA of all films has a maximum at $2-5 \mathrm{~K}$ and decreases with increasing temperature. A large AHA up to $\theta_{\mathrm{H}}=12.7 \%$ is observed at $2 \mathrm{~K}$ for the $80 \mathrm{~nm}$ film and remains high even at room temperature $\left(\theta_{\mathrm{H}}=10.5 \%\right)$. The combined large values of $\sigma_{\mathrm{xy}}^{\mathrm{AHE}}$ and $\theta_{\mathrm{H}}$ in our films suggest an intrinsic mechanism with large Berry curvature in a metallic topological semimetal. The $\sigma_{\mathrm{xy}}^{\mathrm{AHE}}$ and the $\theta_{\mathrm{H}}$ of the $\mathrm{Co}_{2} \mathrm{MnGa}$ films are similar to $\mathrm{Co}_{3} \mathrm{Sn}_{2} \mathrm{~S}_{2},{ }^{49}$ with the gapped nodal line band structure ${ }^{50}$. The anomalous Hall conductivity displays a similar magnitude to metallic films such as $L 1_{0}-\mathrm{FePt},{ }^{51}$ the noncollinear antiferromagnetic Weyl semimetals ${ }^{52,53}$ and the Dirac metal $\mathrm{Fe}_{3} \mathrm{Sn}_{2},{ }^{54}$ however, the $\theta_{\mathrm{H}}$ is an order of magnitude larger, due to the unique topological electronic structure.

\section{CONCLUSIONS}

In summary, we have studied the structural and magnetotransport properties of high-quality magnetron sputtered $\mathrm{Co}_{2} \mathrm{MnGa}$ films with varying thickness. The $\mathrm{Co}_{2} \mathrm{MnGa}$ films are (001)-oriented and almost fullyordered in $L 2_{1}$-type structure. The thinner films show a slight tetragonal distortion, whereas the thicker films approach the perfect cubic structure. Magnetic mea- surements reveal high magnetization and Curie temperature. We find that the magnetotransport properties vary with the temperature and the film thickness. The $80 \mathrm{~nm}$ film shows a large anomalous Hall conductivity up to $1138 \mathrm{~S} / \mathrm{cm}$, accompanied by a large anomalous Hall angle that is maximum at $13 \%$. The strong AHE signal in the $80 \mathrm{~nm}$ thick film agrees very well with bulk firstprinciple calculations with a constant $\gamma$ band broadening approximation, which suggests that the experimentally observed AHE is due to intrinsic mechanism in the bulk limit, and as the thickness decreases the side-jump mechanism has a significant effect. Our work provides a pathway to develop thin film devices that include nodal line and Weyl topological properties intrinsic to the electronic structure.

\section{ACKNOWLEDGMENTS}

This work has been funded by the ERC Advanced Grant No. 291472 "Idea Heusler", ERC Advanced Grant No. 742068 "TOPMAT", EU FET Open RIA Grant No. 766566 "ASPIN", DFG through SFB 1143 (project-id 247310070) and the Würzburg-Dresden Cluster of Excellence on Complexity and Topology in Quantum Matter ct.qmat (EXC 2147, project-id 39085490).
1 A. H. Castro Neto, N. M. R. Peres, K. S. Novoselov, and A. K. Geim, Rev. Mod. Phys. 81, 109 (2009).

2 M. Z. Hasan and C. L. Kane, Rev. Mod. Phys. 82, 3045 (2010).

3 S. M. Young, S. Zaheer, J. C. Y. Teo, C. L. Kane, E. J. Mele, and A. M. Rappe, Phys. Rev. Lett. 108, 1 (2012).

4 Z. Wang, Y. Sun, X.-Q. Chen, C. Franchini, G. Xu, H. Weng, X. Dai, and Z. Fang, Phys. Rev. B 85, 195320 (2012).

5 A. A. Burkov and L. Balents, Phys. Rev. Lett. 107, 127205 (2011).

${ }^{6}$ H. Weng, C. Fang, Z. Fang, B. Andrei Bernevig, and X. Dai, Physical Review X 5, 1 (2015).

7 A. A. Burkov, M. D. Hook, and L. Balents, Phys. Rev. B 84, 235126 (2011).

8 X. Wan, A. M. Turner, A. Vishwanath, and S. Y. Savrasov, Phys. Rev. B 83, 205101 (2011).

${ }^{9}$ Y. Sun, S.-C. Wu, and B. Yan, Phys. Rev. B 92, 115428 (2015).

10 R. Batabyal, N. Morali, N. Avraham, Y. Sun, M. Schmidt, C. Felser, A. Stern, B. Yan, and H. Beidenkopf, Sci. Adv. 2 (2016).

11 H. Inoue, A. Gyenis, Z. Wang, J. Li, S. W. Oh, S. Jiang, N. Ni, B. A. Bernevig, and A. Yazdani, Science 351, 1184 (2016).

12 D. T. Son and B. Z. Spivak, Phys. Rev. B 88, 104412 (2013).

13 X. Huang, L. Zhao, Y. Long, P. Wang, D. Chen, Z. Yang, H. Liang, M. Xue, H. Weng, Z. Fang, X. Dai, and G. Chen, Phys. Rev. X 5, 31023 (2015).
14 C.-L. Zhang, S.-Y. Xu, I. Belopolski, Z. Yuan, Z. Lin, B. Tong, G. Bian, N. Alidoust, C.-C. Lee, S.-M. Huang, T.-R. Chang, G. Chang, C.-H. Hsu, H.-T. Jeng, M. Neupane, D. S. Sanchez, H. Zheng, J. Wang, H. Lin, C. Zhang, H.-Z. Lu, S.-Q. Shen, T. Neupert, M. Zahid Hasan, and S. Jia, Nat. Commun. 7, 10735 (2016).

15 S. A. Parameswaran, T. Grover, D. A. Abanin, D. A. Pesin, and A. Vishwanath, Phys. Rev. X 4, 31035 (2014).

16 T. Higo, H. Man, D. B. Gopman, L. Wu, T. Koretsune, O. M. J. van 't Erve, Y. P. Kabanov, D. Rees, Y. Li, M.T. Suzuki, S. Patankar, M. Ikhlas, C. L. Chien, R. Arita, R. D. Shull, J. Orenstein, and S. Nakatsuji, Nat. Photonics 12, 73 (2018).

17 K. Manna, Y. Sun, L. Muechler, J. Kübler, and C. Felser, Nat. Rev. Mater. 3, 244 (2018), 1802.03771.

18 N. Nagaosa, J. Sinova, S. Onoda, A. H. MacDonald, and N. P. Ong, Rev. Mod. Phys. 82, 1539 (2010).

19 A. A. Burkov, Phys. Rev. Lett. 113, 187202 (2014).

20 J. Noky, J. Gooth, C. Felser, and Y. Sun, Phys. Rev. B 98, 241106 (2018).

21 J. Noky, J. Gayles, C. Felser, and Y. Sun, Phys. Rev. B 97 (2018), 10.1103/PhysRevB.97.220405.

22 S. N. Guin, K. Manna, J. Noky, S. J. Watzman, C. Fu, N. Kumar, W. Schnelle, C. Shekhar, Y. Sun, J. Gooth, and C. Felser, NPG Asia Materials 11, 16 (2019).

23 Z. Wang, M. G. Vergniory, S. Kushwaha, M. Hirschberger, E. V. Chulkov, A. Ernst, N. P. Ong, R. J. Cava, and B. A. Bernevig, Phys. Rev. Lett. 117, 236401 (2016).

24 G. Chang, S.-Y. Xu, H. Zheng, B. Singh, C.-H. Hsu, G. Bian, N. Alidoust, I. Belopolski, D. S. Sanchez, S. Zhang, H. Lin, and M. Z. Hasan, Sci. Rep. 6, 38839 
(2016)

25 J. Kübler and C. Felser, Europhys. Lett. 114, 47005 (2016).

${ }^{26}$ E. Vilanova Vidal, G. Stryganyuk, H. Schneider, C. Felser, and G. Jakob, Appl. Phys. Lett. 99, 132509 (2011).

27 I. Belopolski, D. S. Sanchez, G. Chang, K. Manna, B. Ernst, S.-Y. Xu, S. S. Zhang, H. Zheng, J. Yin, B. Singh, G. Bian, D. Multer, X. Zhou, S.-M. Huang, B. Wang, A. Bansil, H. Lin, C. Felser, and M. Z. Hasan, (2017), arXiv:1712.09992.

28 I. Galanakis, P. H. Dederichs, and N. Papanikolaou, Phys. Rev. B 66, 174429 (2002).

29 P. J. Webster, J. Phys. Chem. Solids 32, 1221 (1971).

30 P. J. Brown, K. U. Neumann, P. J. Webster, and K. R. A. Ziebeck, J. Phys.: Condens. Matter 12, 1827 (2000).

31 K. Manna, L. Muechler, T.-H. Kao, R. Stinshoff, Y. Zhang, J. Gooth, N. Kumar, G. Kreiner, K. Koepernik, R. Car, J. Kübler, G. H. Fecher, C. Shekhar, Y. Sun, and C. Felser, Phys. Rev. X 8, 41045 (2018).

32 A. Sakai, Y. P. Mizuta, A. A. Nugroho, R. Sihombing, T. Koretsune, M.-T. Suzuki, N. Takemori, R. Ishii, D. Nishio-Hamane, R. Arita, P. Goswami, and S. Nakatsuji, Nat. Phys. 14, 1119 (2018).

${ }^{33}$ G. Chang, S.-Y. Xu, X. Zhou, S.-M. Huang, B. Singh, B. Wang, I. Belopolski, J. Yin, S. Zhang, A. Bansil, H. Lin, and M. Z. Hasan, Phys. Rev. Lett. 119, 156401 (2017).

34 B. M. Ludbrook, B. J. Ruck, and S. Granville, Appl. Phys. Lett. 110, 62408 (2017).

${ }^{35}$ H. Reichlova, R. Schlitz, S. Beckert, P. Swekis, A. Markou, Y.-C. Chen, D. Kriegner, S. Fabretti, G. Hyeon Park, A. Niemann, S. Sudheendra, A. Thomas, K. Nielsch, C. Felser, and S. T. B. Goennenwein, Appl. Phys. Lett. 113, 212405 (2018).

${ }^{36}$ D. Kriegner, E. Wintersberger, and J. Stangl, J. Appl. Cryst. 46, 1162 (2013).

37 U. Pietsch, V. Holy, and T. Baumbach, High-resolution $X$-ray scattering: from thin films to lateral nanostructures (Springer New York, 2004).
${ }^{38}$ K. Özdoğan, E. Şaşığlu, B. Aktaş, and I. Galanakis, Phys. Rev. B 74, 172412 (2006).

39 D. A. Goodings, Phys. Rev. 132, 542 (1963).

40 L. J. Zhu and J. H. Zhao, Sci. Rep. 7, 42931 (2017).

41 E. Vilanova Vidal, H. Schneider, and G. Jakob, Phys. Rev. B 83, 174410 (2011).

42 M. S. Gabor, M. Belmeguenai, T. Petrisor, C. UlhaqBouillet, S. Colis, and C. Tiusan, Phys. Rev. B 92, 54433 (2015).

43 G. Kresse and J. Furthmüller, Phys. Rev. B 54, 11169 (1996).

44 R. Kubo, J. Phys Soc. Jpn. 12, 570 (1957).

45 S. Lowitzer, D. Ködderitzsch, and H. Ebert, Phys. Rev. Lett. 105, 266604 (2010).

46 A. Kovalev, J. Sinova, and Y. Tserkovnyak, Phys. Rev. Lett. 105, 36601 (2010).

47 J. Weischenberg, F. Freimuth, J. Sinova, S. Blügel, and Y. Mokrousov, Phys. Rev. Lett. 107, 106601 (2011).

48 C. Gorini, U. Eckern, and R. Raimondi, Phys. Rev. Lett. 115, 076602 (2015).

${ }^{49}$ E. Liu, Y. Sun, N. Kumar, L. Muechler, A. Sun, L. Jiao, S.-Y. Yang, D. Liu, A. Liang, Q. Xu, J. Kroder, V. Süß, H. Borrmann, C. Shekhar, Z. Wang, C. Xi, W. Wang, W. Schnelle, S. Wirth, Y. Chen, S. T. B. Goennenwein, and C. Felser, Nat. Phys. 14, 1125 (2018).

50 Q. Xu, E. Liu, W. Shi, L. Muechler, J. Gayles, C. Felser, and Y. Sun, Phys. Rev. B 97, 235416 (2018).

51 P. B. Allen, H. Berger, O. Chauvet, L. Forro, T. Jarlborg, A. Junod, B. Revaz, and G. Santi, Phys. Rev. B 53, 4393 (1996).

52 S. Nakatsuji, N. Kiyohara, and T. Higo, Nature 527, 212 (2015).

53 A. K. Nayak, J. E. Fischer, Y. Sun, B. Yan, J. Karel, A. C. Komarek, C. Shekhar, N. Kumar, W. Schnelle, J. Kübler, C. Felser, and S. S. P. Parkin, Sci. Adv. 2, e1501870 (2016).

54 L. Ye, M. Kang, J. Liu, F. von Cube, C. R. Wicker, T. Suzuki, C. Jozwiak, A. Bostwick, E. Rotenberg, D. C. Bell, L. Fu, R. Comin, and J. G. Checkelsky, Nature 555, 638 (2018). 